\title{
Fatigue in patients with inflammatory bowel disease is associated with distinct differences in immune parameters
}

This article was published in the following Dove Press journal:

Clinical and Experimental Gastroenterology

2 May 2017

Number of times this article has been viewed

\section{Lauran Vogelaar ${ }^{1, *}$ \\ Colin de Haar ${ }^{2, *}$ \\ Bas RJ Aerts' \\ Maikel P Peppelenbosch' \\ Reinier Timman ${ }^{3}$ \\ Bettina E Hanssen' \\ C Janneke van der Woude'}

'Department of Gastroenterology and Hepatology, Erasmus MC, Rotterdam,

${ }^{2}$ Applied Tumor Immunology,

Laboratory of Translational

Immunology, UMC Utrecht,

Utrecht, ${ }^{3}$ Department of Psychiatry,

Section of Medical Psychology

and Psychotherapy, Erasmus MC,

Rotterdam, the Netherlands

*These authors contributed equally to this work
Correspondence: Lauran Vogelaar Department of Gastroenterology and Hepatology, Erasmus MC, 's Gravendijkwal 230, Room HS 306, 3000

CA Rotterdam, the Netherlands

Email l.vogelaar@erasmusmc.nl
Background: Although it is well recognized that fatigue is an important problem in many of the quiescent inflammatory bowel disease (IBD) patients, it is unknown whether the immune status is different in fatigued versus non-fatigued patients. In this study, we contrasted various characteristics of the immune system in fatigued against non-fatigued patients with IBD in clinical remission.

Patients and methods: Patients with IBD in clinical remission were phenotyped according to the Montreal classification, and the checklist individual strength-fatigue (CIS-fatigue) was used to assess fatigue (CIS-fatigue $\geq 35$ ). Flow cytometry on peripheral blood samples was used to investigate differences in leukocyte subsets. The expression of various cytokines was determined in stimulated whole blood and serum samples using enzyme-linked immunosorbent assay. Differences between fatigued and non-fatigued patients with IBD were assessed.

Results: In total, 55 patients were included in the fatigue group (FG) and 29 patients in the non-fatigue group (NFG). No differences in demographic and clinical characteristics were observed between the groups. Flow cytometry data showed a significantly lower percentage of monocytes $(p=0.011)$ and a higher percentage of memory T-cells $(p=0.005)$ and neutrophils $(p=0.033)$ in the FG compared with the NFG. Whole blood stimulation showed increased TNF- $\alpha(p=0.022)$ and IFN- $\gamma(p=0.047)$ in the FG. The median serum level was significantly higher for IL-12 $(p<0.001)$ and IL-10 $(p=0.005)$ and lower for IL-6 $(p=0.002)$ in the FG compared with NFG.

Conclusion: Significant differences in immune profile between fatigued and non-fatigued patients with IBD in clinical remission were found, which point out to a chronically active and Th1-skewed immune system in patients with fatigue. Whether these immune differences are directly involved in the fatigue complaints via immune-to-brain communication pathways remains to be determined. As such, further exploration of the underlying immune effects associated with fatigue is warranted to determine potential treatment options.

Keywords: inflammatory bowel disease, fatigue, leukocyte subsets, cytokines

\section{Introduction}

Patients with inflammatory bowel disease (IBD) suffer from an immune-mediated chronic relapsing disease. This disease significantly impairs the health-related quality of life of patients. Fatigue is an important factor negatively affecting the health-related quality of life in these patients. ${ }^{1-4}$ With $>40 \%$ of patients with IBD suffering from fatigue, even when the disease is in remission, further understanding of the etiology of fatigue in IBD is warranted. ${ }^{1,3,5}$ 
There is bidirectional communication between the brain and the immune system.

Activation of the immune system could lead to the release of proinflammatory cytokines that act on the brain to induce sickness behavior (including fatigue) or perhaps even lead to depression in vulnerable individuals. ${ }^{6}$ In addition, the brain is able to affect the immune system directly by means of neurotransmitters from either the sympathetic or parasympathetic neurons or indirectly via the induction of hormones such as cortisol.?

As such, fatigue can affect the immune system and vice versa. If fatigue influences the immune system in IBD, it is well possible that stratification for pharmacological treatment based on fatigue status may improve clinical outcome. If fatigue merely reflects ongoing immune activation in patients with IBD, it might also implicate the potential usefulness of targeted therapy for IBD-related fatigue.

A variety of factors, including disease activity, female sex, psychological well-being, medication use, anemia and sleep difficulties, are known to influence the severity of fatigue in patients with IBD, and interestingly, many of these factors may in turn also affect immune parameters. ${ }^{3,4,8}$ Indeed, in other diseases, especially chronic fatigue syndrome (CFS), fatigue and immunity show important correlations..$^{9-15}$ The mechanisms by which fatigue status is linked to the immune system remain largely obscure, but in view of the increasing evidence for the existence of a gut-brain axis, it is well possible that in IBD also, fatigue-related effect of the immune system may exist. ${ }^{16-18}$ This effect of the immune system was also proposed in fatigued multiple sclerosis and patients with cancer, where higher levels of proinflammatory cytokines were seen in fatigued patients..$^{19,20}$

The observation that in patients with cancer fatigue may persist for years after treatment completion in otherwise healthy individuals is of special interest since it may reflect the remission situation in patients with IBD with regard to disease activity. ${ }^{21,22}$ In patients post cancer treatment, elevated levels of markers of inflammation were found in the circulation (IL-1ra, sTNF-RII and IL-6R) and in peripheral blood mononuclear cells (PBMCs) stimulated with lipopolysaccharide (LPS; IL-1b, IL-6 and TNF- $\alpha$ ). ${ }^{23}$

Nevertheless, the relation between fatigue status and immune status in IBD remains unexplored, and thus studies on this aspect of IBD are urgently called for. The abovementioned considerations prompted us to investigate whether fatigued patients with IBD differ from non-fatigued patients with IBD with respect to immunity.

\section{Patients and methods Study design}

For this study, we exploited an earlier published study cohort in which the effects of solution-focused therapy on fatigue in patients with IBD were characterized. ${ }^{24}$

The use of this cohort allowed us to include patients with well-characterized fatigue status. Patients from this cohort were asked to participate in this study.

The checklist individual strength (CIS) was used to determine whether a patient suffered from fatigue (CIS-fatigue subscale score $\geq 35) .{ }^{25}$

The CIS is a 20 -item patient-reported validated instrument measuring severity of fatigue, motivation, activity level and concentration. The severity of fatigue, measured with the subscale "fatigue", was used as an outcome measure. Patients with a score of $\geq 35$ on this subscale were considered to be fatigued. The CIS is a renewed format of the multifactorial fatigue index (MFI), where five questions are differently formulated and all other questions are the same. The CIS is standardized and uses a cut-off score for fatigue in contrast to the MFI.

As described in our earlier published study cohort, fecal calprotectin concentration was measured in the fatigued patients. Levels of $<200 \mathrm{mg} / \mathrm{g}$ were regarded as compatible with disease remission. ${ }^{24}$ The Harvey-Bradshaw index (HBI) $<5$ for Crohn's disease or the colitis activity index $(\mathrm{CAI})<10$ for ulcerative colitis was used to determine clinical remission of the disease in the non-fatigued patients..$^{26,27}$

Demographics and disease phenotype (Montreal classification) were collected from medical records, and concomitant medication use was investigated using a questionnaire focusing on current medication use and subjective side effects to medication. $^{28}$

After these baseline measurements and measurements on blood samples, the fatigue group (FG) was enrolled in a clinical trial to study the effects of psychotherapy, especially solution-focused therapy on fatigue of which the results have been described. ${ }^{24}$ Consecutive patients with IBD from the same hospitals as the fatigue cohort, with a CIS-fatigue score $<35$, were enrolled in the non-fatigue group (NFG) for this study. As in the FG, patients of the NFG were aged $\geq 18$ years, and the diagnosis of IBD was radiologically or endoscopically/histologically confirmed. Exclusion criteria, as described in the clinical trial, were equal for the FG and NFG.

Differences in baseline measurements between the FG and the NGF included laboratory values. In the NFG patients, only C-reactive protein (CRP) and leukocytes were measured. 
This study was conducted in accordance with the protocol International Conference on Harmonization Guidelines for Good Clinical Practice, the Declaration of Helsinki and local national regulations governing clinical study conduct and was registered at the medical ethical committee (MEC) of the Erasmus Medical Center (registration number: MEC2010-107; NL32020.078.10). The protocol was approved by the institutional review board (MEC) of the Erasmus Medical Center. All patients gave written informed consent. Patients were enrolled in the Netherlands from January 2010 to January 2011 by the principal investigator.

\section{Blood collection and stimulation}

Following blood drawing, within 24 hours serum was obtained using a coagulation tube and stored at $-80^{\circ} \mathrm{C}$ until further analysis. Serum was collected from both fatigued and non-fatigued patients with IBD, and immune assays were conducted at the same location for all blood samples.

Heparinized whole blood samples were diluted 1:10 with Roswell Park Memorial Institute (RPMI) 1640 (Lonza, Basel, Switzerland) and were stimulated with $25 \mu \mathrm{g} / \mathrm{mL}$ phytohemagglutinin (PHA; Remel, Lenexa, KS, USA) or $100 \mathrm{ng} / \mathrm{mL}$ LPS (Sigma-Aldrich, Zwijndrecht, the Netherlands). Supernatants of the LPS- and PHA-stimulated cultures were obtained at 24 and 72 hours, respectively, and stored at $-80^{\circ} \mathrm{C}$ until further analysis.

\section{Leukocyte subsets analysis}

After removal of erythrocytes using ery-lysis buffer, the heparinized whole blood samples were stained using antibodies against CD16 (Pacific Blue), CD14 (PerCP/ Cy5.5), CD56 (PE/Cy7) and CD62-L (Alexa Fluor 647) purchased from BioLegend (San Diego, CA, USA); CD3 (AmCyan) and CD4 (APC-H7) purchased from BD Biosciences (Franklin Lakes, NJ, USA); CD45RA (FITC) purchased from eBioscience (San Diego, CA, USA) and CD19 (PE) purchased from Beckman Coulter (Brea, CA, USA) to analyze the different leukocyte subsets using the FACSCanto II flow cytometer with FACSDiva software from BD Biosciences. The different leukocyte subsets were identified and counted using FlowJo software (Tree Star, Inc., Ashland, OR, USA).

The leukocytes were subdivided into three main populations based on forward scatter and side scatter: lymphocytes, granulocytes and monocytes. The different leukocyte subsets were subdivided into lymphocytes (T-cells, B-cells, cytotoxic T-cells, T-helper cells, memory T-cells, effector T-cells, naïve T-cells and NK-cells), monocytes (CD14+CD16+, CD14-
CD16+ and CD14+CD16-) and granulocytes (eosinophils and neutrophils) as shown in Table 1.

\section{Cytokine levels}

Serum and supernatant levels of IL-4, IL-5, IL-6, IL-8, IL-10, IL-12, TNF- $\alpha$ and IFN- $\gamma$ were assessed using Ready-Set-Go! $®$ enzyme-linked immunosorbent assay sets from eBioscience according to the manufacturer's instructions and using MaxiSorp 96-well plates (Nunc; Thermo Fisher Scientific, Waltham, MA, USA) and a model 680 microplate reader from Bio-Rad (Hercules, CA, USA). When levels were below the detection limit of 2.0, these were considered left-hand censored.

\section{Statistical analysis}

For differences in characteristics and disease phenotypes between the FG and NFG, $\chi^{2}$ tests were used for dichotomous variables and $t$-tests for continuous variables.

Normality of laboratory parameters, leukocyte subsets and cytokines were determined using Shapiro-Wilk tests. All outcomes, except lymphocytes, granulocytes, monocytes, naïve T-cells and memory T-cells, were not normally distributed. Differences between the FG and NFG were analyzed with $t$-tests for normally distributed outcomes and with Mann-Whitney $U$ tests for abnormally distributed outcomes. A number of laboratory parameters for serum cytokines were below the detectable range of 2.0. For these parameters, we performed Tobit analyses for low-censored data on the ranktransformed variables. ${ }^{29}$ Median values, interquartile ranges and differences are presented in Table 1.

Tobit analyses were performed with Stata version 13.1 (SataCorp LP, College Station, TX, USA). All other analyses were performed with SPSS software for Windows, V.20 (SPSS, Chicago, IL, USA).

Results were considered significant when two-sided $p$-values were $<0.05$; no correction for multiple testing was applied because of the exploratory nature of the study.

\section{Results \\ Patient characteristics}

In total, 55 fatigued patients with IBD (FG) of the earlier published study cohort agreed to participate in this study, and 29 patients in the NFG were included.

Before analyzing the differences in immune parameters, the demographic and clinical characteristics of the groups were analyzed (FG vs. NFG; Tables 2 and 3). No differences were observed between the two groups. With regard to remission of the disease, the NFG showed a mean CAI score of 2.3 (standard deviation [sd] 0.6) and an HBI score of 1.2 (sd 1.1). 
Table I Leukocyte subsets and cytokines

\begin{tabular}{|c|c|c|c|c|c|}
\hline \multirow[t]{2}{*}{ Baseline } & \multicolumn{2}{|c|}{ Fatigue $(n=55)$} & \multicolumn{2}{|c|}{ Non-fatigue $(n=29)$} & \multirow[t]{2}{*}{$p$-value } \\
\hline & Median & [Interquartile range] & Median & [Interquartile range] & \\
\hline Lymphocytes & 33.2 & {$[22.8-46.0]$} & 35.0 & {$[28.7-50.7]$} & 0.188 \\
\hline NK-cells & 2.7 & {$[0.4-6.6]$} & 4.7 & [0.9-8.2] & 0.171 \\
\hline B-cells & 8.5 & {$[4.7-11.2]$} & 8.6 & {$[4.4-12.4]$} & 0.158 \\
\hline T-cells & 78.3 & {$[59.5-82.4]$} & 77.8 & {$[66.2-82.7]$} & 0.963 \\
\hline Leukocytes & 6.0 & {$[5.1-7.6]$} & 5.4 & {$[4.5-7.2]$} & 0.862 \\
\hline T-helper cells & 64.4 & {$[57.4-71.3]$} & 67.6 & {$[54.3-74.8]$} & 0.519 \\
\hline Naïve T-cells & 36.1 & {$[26.5-43.0]$} & 47.0 & {$[29.2-58.1]$} & 0.062 \\
\hline Memory T-cells* & 43.7 & [36.4-49.9] & 33.7 & {$[27.9-39.6]$} & 0.005 \\
\hline Effector T-cells & 16.5 & {$[10.3-23.6]$} & 12.8 & {$[8.2-23.0]$} & 0.263 \\
\hline Cytotoxic T-cells & 35.6 & {$[28.7-42.6]$} & 32.4 & {$[25.2-45.7]$} & 0.527 \\
\hline Granulocytes & 46.6 & {$[34.2-58.6]$} & 40.5 & {$[28.5-52.6]$} & 0.351 \\
\hline Eosinophils & 3.6 & {$[2.5-6.7]$} & 4.9 & {$[2.7-|| .8]$} & 0.097 \\
\hline Neutrophils* & 77.0 & [64.6-83.6] & 68.8 & [58.9-78.2] & 0.033 \\
\hline Monocytes* & 5.3 & {$[3.5-7.5]$} & 7.2 & [4.7-9.1] & 0.011 \\
\hline CDI4-CDI6+* & 7.9 & {$[5.7-|| .5]$} & 11.9 & {$[7.9-15.3]$} & 0.017 \\
\hline CDI4+ CDI6+* & 3.5 & {$[2.8-6.6]$} & 2.6 & {$[2.1-4.0]$} & 0.010 \\
\hline CDI4+ CDI6- & 86.9 & {$[82.2-91.3]$} & 84.6 & [80.8-90.5] & $0.36 \mathrm{I}$ \\
\hline \multicolumn{6}{|l|}{ PHA-stimulated WB } \\
\hline IL-5 & 118.1 & {$[86.5-2 \mid 1.2]$} & 85.6 & {$[62.3-148.7]$} & 0.078 \\
\hline IL-6 & 2452 & [1629-13758] & 3464 & [1224-15218] & 0.982 \\
\hline IL-8 & 57143 & [4I054-94I30] & 46237 & [23679-85|22] & 0.156 \\
\hline IL-10 & 658 & {$[326-1108]$} & 276 & {$[154-1133]$} & 0.132 \\
\hline TNF- $\alpha^{*}$ & 224 & {$[112-678]$} & 125 & [48-438] & 0.022 \\
\hline IFN-g* & 28875 & {$[|7487-5| 398]$} & 9536 & {$[2525-75476]$} & 0.047 \\
\hline \multicolumn{6}{|l|}{ LPS-stimulated WB } \\
\hline IL-6 & 3115 & [2394-5276] & 5065 & [3287-7268] & 0.046 \\
\hline IL-8 & 5844 & [2816-9539] & 4760 & [3067-1064I] & 0.777 \\
\hline IL-10 & 200 & [107-304] & 136 & [96-275] & 0.651 \\
\hline TNF- $\alpha$ & 560 & {$[174-1208]$} & 206 & [66-709] & 0.073 \\
\hline \multicolumn{6}{|c|}{ Serum cytokines $(\%$ below } \\
\hline \multicolumn{6}{|c|}{ detectable range in FG and NFG) } \\
\hline IL-4 (64\%-7I\%) & 2.0 & {$[2.0-3.2]$} & 2.0 & {$[2.0-2.4]$} & 0.570 \\
\hline IL-5 (19\%-25\%) & 14.7 & {$[3.2-54.0]$} & 10.0 & {$[2.0-27.6]$} & 0.269 \\
\hline IL-6* $(83 \%-42 \%)$ & 2.0 & {$[2.0-2.0]$} & 2.3 & {$[2.0-4.7]$} & 0.002 \\
\hline IL-8 (28\%-29\%) & 2.6 & {$[2.0-4.8]$} & 5.7 & {$[2.0-50.7]$} & 0.122 \\
\hline IL- $10 *(42 \%-67 \%)$ & 2.2 & {$[2.0-3.1]$} & 2.0 & {$[2.0-2.4]$} & 0.005 \\
\hline IL-I $2 *(4 \%-33 \%)$ & 4.8 & {$[3.8-9.0]$} & 3.3 & {$[2.0-4.4]$} & $<0.001$ \\
\hline TNF- $\alpha(49 \%-4 \%)$ & 49.5 & [11.9-94.1] & 45.1 & {$[13.5-174.2]$} & 0.498 \\
\hline IFN-g (I5\%-29\%) & 4.6 & {$[2.8-7.3]$} & 3.3 & {$[2.0-10.8]$} & 0.293 \\
\hline
\end{tabular}

Notes: Unpaired two samples t-test for normally distributed variables (lymphocytes, granulocytes, monocytes, naïve T-cells and memory T-cells). Mann-Whitney $U$ test for not normally distributed variables. Tobit test for serum cytokines with low-censored values. $* p<0.05$. Lymphocytes, granulocytes and monocytes: percentage of whole blood. CDI4+ CDI6+ and CDI4+ CDI6- monocytes: percentage of total monocytes. T-helper cells and cytotoxic T-cells: percentage of T-cells. Naïve T-cells, memory T-cells and effector T-cells: percentage of T-helper cells. Eosinophils and neutrophils: percentage of granulocytes. CDI4- CDI6+, CDI4+ CDI6+ and CDI4+ CDI6- monocytes: percentage of total monocytes. Cytokine concentrations are in $\mathrm{pg} / \mathrm{mL}$.

Abbreviations: $n$, number of patients; PHA, phytohemagglutinin; WB, whole blood; LPS, lipopolysaccharide; FG, fatigue group; NFG, non-fatigue group.

The mean calprotectin level in the FG was $66 \mu \mathrm{g} / \mathrm{g}$. Based on these scores, both groups were in clinical remission.

\section{Whole blood leukocytes}

As a first crude indicator of a link between fatigue status and the immune system in patients with IBD, the composition of the leukocyte system was investigated using flow cytometry. No differences were detected in total leukocyte numbers (Table 1) between the groups. Within the major leukocyte subpopulations (lymphocytes, granulocytes and monocytes), a significant lower percentage of monocytes (median: FG: 5.3, NFG: $7.2 ; p=0.011$ ) was detected in the FG compared with the NFG. When monocytes were further subphenotyped, we found a significant lower percentage of the non-classical CD14 ${ }^{\text {dim }} \mathrm{CD} 16^{+}$monocytes in the $\mathrm{FG}$ compared with the NFG (median: FG: 7.9, NFG: 11.9; $p=0.017$ ).

Within the lymphocyte subsets, no differences were detected; only within the CD4+ (helper) T-cell population, 
Table 2 Patient characteristics

\begin{tabular}{|c|c|c|c|}
\hline Patient characteristics & $\begin{array}{l}\text { Fatigue } \\
(n=55)\end{array}$ & $\begin{array}{l}\text { Non-fatigue } \\
(n=29)\end{array}$ & p-value \\
\hline Age in years; mean (sd) & $40.1(10.4)$ & $40.7(14.4)$ & 0.861 \\
\hline Females, n (\%) & $36(65 \%)$ & $13(45 \%)$ & 0.068 \\
\hline Crohn's disease, n (\%) & $42(76 \%)$ & $26(90 \%)$ & 0.140 \\
\hline Ulcerative Colitis, n (\%) & $13(24 \%)$ & $3(10 \%)$ & \\
\hline \multicolumn{4}{|c|}{ Current medication use, $\mathrm{n}(\%)$} \\
\hline 5-ASA & $20(36 \%)$ & $10(34 \%)$ & 0.954 \\
\hline Immunosuppressives & $20(36 \%)$ & $13(45 \%)$ & 0.376 \\
\hline Corticosteroids & $10(18 \%)$ & $5(17 \%)$ & 0.971 \\
\hline Biologicals (anti-TNF) & $13(24 \%)$ & $9(31 \%)$ & 0.406 \\
\hline \multicolumn{4}{|c|}{ Side effects to medication, $\mathrm{n}(\%)$} \\
\hline 5-ASA & $3(5 \%)$ & I (3\%) & 0.484 \\
\hline Immunosuppressives & II (20\%) & 7 (24\%) & 0.402 \\
\hline Corticosteroids & $3(5 \%)$ & $3(10 \%)$ & 0.876 \\
\hline Biologicals (anti-TNF) & $8(15 \%)$ & $3(10 \%)$ & 0.066 \\
\hline \multicolumn{4}{|l|}{ Disease activity, $\mathrm{n}$} \\
\hline CAI & 14, 4 (mean) & 4, 2.3 (mean) & 0.805 \\
\hline $\mathrm{HBI}$ & 4I, 2.8 (mean) & $25,1.2$ (mean) & 0.035 \\
\hline
\end{tabular}

Notes: Chi-square test for dichotomous variables and $t$-test for continuous variables. Corticosteroids: prednisone and budesonide. Immunosuppressives: azathioprine, methotrexate and cyclosporine.

Abbreviations: $n$, number of patients; sd, standard deviation; 5-ASA, 5-aminosalicylic acid; CAl, colitis activity index; $\mathrm{HBI}$, Harvey-Bradshaw index.

a significantly higher percentage of central memory CD4+ T cells (median: FG: 43.7, NFG: $33.7 ; p=0.005$ ) in the FG compared with the NFG was found. Further analysis of the granulocyte population showed a significantly higher percentage of neutrophils (median: FG: 77.0, NFG: 68.8; $p=0.033$ ) in the FG compared with the NFG.

\section{Whole blood cytokine production}

In addition to the leukocyte subset analysis, we also determined the production of cytokines by the leukocytes after stimulation with PHA or LPS, in which PHA stimulates mostly lymphocytes and LPS is more prone to trigger the innate granulocytes and monocytes to produce cytokines (Table 1). PHA stimulation induced higher median cytokine levels in whole blood from the FG for all cytokines measured except for IL-6. Of these cytokines, the levels of TNF- $\alpha$ (median: FG: 224, NFG: $125 ; p=0.022$ ) and IFN- $\gamma$ (median: FG: 28875, NFG: 9536; $p=0.047$ ) were significantly higher in the FG compared with the NFG. LPS stimulation induced significantly higher median IL-6 levels in the NFG compared with the FG (median: FG: 3114, NFG: 5064; $p=0.046$ )

\section{Serum cytokine levels}

We also investigated the serum levels of a variety of cytokines (Table 1). The levels of IL-12 (median: FG: 4.8, NFG: $3.3 ; p$ $<0.001$ ) and IL-10 (median: FG: 2.2, NFG: 2.0 [lower limit]; $p=0.005$ ) were significantly higher in the FG serum samples,
Table 3 Disease phenotype

\begin{tabular}{|c|c|c|c|}
\hline Montreal classification & $\begin{array}{l}\text { Fatigue } \\
(n=55)\end{array}$ & $\begin{array}{l}\text { Non-fatigue } \\
(n=29)\end{array}$ & $p$-value \\
\hline \multicolumn{4}{|l|}{ Montreal classification - $\mathrm{CD}^{28}$} \\
\hline Mean age at diagnosis in yrs (sd) & $27.4(9.2)$ & $24.0(9.0)$ & 0.135 \\
\hline \multicolumn{4}{|l|}{ Age at diagnosis, $\mathrm{n}(\%)$} \\
\hline $\mathrm{Al}$ & $4(9.8)$ & $7(26.9)$ & 0.065 \\
\hline $\mathrm{A} 2$ & $31(75.6)$ & $18(69.2)$ & 0.566 \\
\hline $\mathrm{A} 3$ & $6(14.6)$ & I (3.8) & 0.159 \\
\hline \multicolumn{4}{|l|}{ Location } \\
\hline LI & $4(9.8)$ & $3(11.5)$ & 0.816 \\
\hline L2 & $17(4 \mid .5)$ & $9(34.6)$ & 0.575 \\
\hline L3 & $20(48.8)$ & $13(50.0)$ & 0.922 \\
\hline L4 & I (2.4) & I (3.8) & 0.742 \\
\hline$+\mathrm{L} 4$ & $40(2.4)$ & $23(11.5)$ & 0.126 \\
\hline \multicolumn{4}{|l|}{ Behavior } \\
\hline $\mathrm{BI}$ & $28(68.3)$ & $15(57.7)$ & 0.378 \\
\hline B2 & $6(14.6)$ & $6(23.1)$ & 0.380 \\
\hline B3 & $6(14.6)$ & $5(19.2)$ & 0.621 \\
\hline$p$ & $30(26.8)$ & $17(34.6)$ & 0.497 \\
\hline \multicolumn{4}{|l|}{ Surgery $C D$} \\
\hline Bowel resection (\%) & 46.3 & 61.5 & 0.225 \\
\hline Number of resections; mean (sd) & $\mathrm{I}(0.9)$ & $\mathrm{I} .5(\mathrm{I} . \mathrm{I})$ & 0.122 \\
\hline Age at first resection; mean (sd) & $29.8(9.7)$ & $29.5(13.1)$ & 0.930 \\
\hline Stoma (\%) & 14.6 & 15.4 & 0.933 \\
\hline Rectum amputation (\%) & 7.3 & 7.7 & 0.955 \\
\hline \multicolumn{4}{|l|}{ Montreal classification - UC ${ }^{28}$} \\
\hline Mean age at diagnosis in yrs (sd) & $29.2(8.1)$ & $35.7(6.0)$ & 0.215 \\
\hline \multicolumn{4}{|l|}{ Age at diagnosis, $n(\%)$} \\
\hline $\mathrm{Al}$ & $0(0)$ & $0(0)$ & \\
\hline $\mathrm{A} 2$ & $13(92.3)$ & $2(66.7)$ & 0.226 \\
\hline $\mathrm{A} 3$ & I (7.7) & I (33.3) & 0.226 \\
\hline \multicolumn{4}{|l|}{ Location } \\
\hline $\mathrm{EI}$ & I (7.7) & $0(0)$ & 0.620 \\
\hline E2 & $8(53.8)$ & $3(100)$ & 0.137 \\
\hline E3 & $5(38.5)$ & $0(0)$ & 0.195 \\
\hline \multicolumn{4}{|l|}{ Severity } \\
\hline so & $7(46.2)$ & $0(0)$ & 0.137 \\
\hline SI & $7(53.8)$ & $2(66.7)$ & 0.687 \\
\hline S2 & $0(0)$ & I (33.3) & 0.032 \\
\hline S3 & $0(0)$ & $0(0)$ & \\
\hline \multicolumn{4}{|l|}{ Surgery UC } \\
\hline Bowel resection (\%) & 7.1 & 0 & 0.633 \\
\hline Number of resections, mean & 1 & 0 & - \\
\hline Age at first resection, mean & 22 & - & - \\
\hline Stoma (\%) & 0 & 0 & - \\
\hline Rectum amputation (\%) & 0 & 0 & - \\
\hline \multicolumn{4}{|l|}{ Laboratory CD and UC } \\
\hline $\begin{array}{l}\text { CRP; median [interquartile } \\
\text { range] - baseline }\end{array}$ & $\begin{array}{l}1.0 \\
{[0.0-2.0]}\end{array}$ & $2.0[1.0-3.0]$ & 0.140 \\
\hline $\begin{array}{l}\text { Leukocytes; median } \\
\text { [interquartile range] - baseline }\end{array}$ & $\begin{array}{l}6.0 \\
{[5.1-7.6]}\end{array}$ & $5.4[4.5-7.2]$ & 0.188 \\
\hline
\end{tabular}

Notes: Chi-square test for dichotomous variables. Mann-Whitney U-test for the continuous variables, CRP and leukocytes. $t$-test for all other continuous variables. Abbreviations: $n$, number of patients; $C D$, Crohn's disease; yrs, years; sd, standard deviation; UC, ulcerative colitis; CRP, C-reactive protein.

whereas the levels of IL-6 were significantly reduced in the FG serum compared with the NFG serum (median: FG: 2.0 [lower detection limit], NFG: $2.3 ; p=0.002)$. 


\section{Discussion}

A large portion of patients with IBD suffer from fatigue even when the disease is in clinical remission. The intricate bidirectional relation between the immune system and the brain justifies the search for possible difference in immune parameters in these patients.

Activation of the immune system could lead to the release of proinflammatory cytokines that act on the brain to induce sickness behavior (including fatigue) or maybe even lead to depression in vulnerable individuals. ${ }^{6}$ In addition, the brain is able to affect the immune system directly by means of neurotransmitters from either the sympathetic or parasympathetic neurons or indirectly via the induction of hormones such as cortisol. ${ }^{7}$

We compared a large variety of immune parameters between fatigued and non-fatigued patients with IBD to determine whether there were parameters that were discriminative between the groups.

Since we are the first to compare a large variety of immune parameters between patients with IBD with fatigue and without fatigue, we can only mirror our data to those studies that assessed immune parameters in patients suffering from fatigue in different disease settings such as cancer, CFS and chronic viral infections. ${ }^{9-15,30-36}$

Since viral infections are a popular proposed cause of CFS, it was interesting to notice that naïve CD4 cells and enhanced memory cells are a sign of chronic adaptive immune activation and have been previously reported in patients with chronic hepatitis $\mathrm{C}$ infection and were associated with cytomegalovirus and Helicobacter pylori titers. ${ }^{37-39}$ As such, these data support the idea that there may still be some ongoing immune activation of unknown origin involved in the fatigue complaints. Indeed, fatigued cancer survivors showed a $31 \%$ increase in circulating $\mathrm{T}$ lymphocytes relative to non-fatigued controls, particularly CD4+ T lymphocytes ( $41 \%$ increase) and CD56+ effector T lymphocytes (52\% increase).

As with the T-cell changes, differences in neutrophils and monocytes between fatigued and non-fatigued patients could all be pointing toward an ongoing infection in the fatigued patients with IBD.

Both the significantly enhanced TNF- $\alpha$ and IFN- $\gamma$ release upon stimulation with PHA are in line with observations in CFS patients where PBMCs instead of whole blood were stimulated. ${ }^{40}$ Both of them are also supportive of a Th1skewed immunity driving the fatigue complaints in patients with IBD.

With regard to the serum proinflammatory cytokine levels, no significant difference in the levels of TNF- $\alpha$ was observed as often reported in CFS..$^{41,42}$ However, anti-TNF treatment could influence these results.

The enhanced IL-12 serum levels in fatigued patients with IBD were also in line with data previously reported on plasma of CFS patients compared to healthy controls..$^{10}$ Although the reduced IL-6 levels were in contrast with this study, reduced IL-6 levels were reported in a recent study when moderate CFS patients were compared with healthy controls. ${ }^{10,43}$

The differences in immune parameters between the fatigued and non-fatigued patients with IBD suggest that there is a link between the immune system and the brain in IBD-associated fatigue. Whether this is a direct link cannot be concluded from our data.

Most of our data support the hypothesis that there is an ongoing low level of immune activation in patients with IBD who present fatigue complaints while in clinical remission. Because of the multifactorial origins of fatigue, there could be a variety of causes of the observed immune activation that may even differ between patients presenting similar immune parameters. Viral infections either acute or chronic may be involved in the IBD-associated fatigue. ${ }^{38,44}$ Since serology status for different viral infections was not obtained during this study, we cannot rule out their possible role in at least subsets of the fatigued patients. Another possible cause may be the occurrence of microscopic relapses, at the level of the lamina propria, without affecting clinical disease symptoms. ${ }^{45}$ Since our patients were in clinical remission, no biopsies are available to rule out this possibility. Microscopic disease activity may enable enhanced translocation of bacteria, described as "leaky gut", a phenomenon that has been associated with CFS as well. ${ }^{46,47}$

Since microbes are well known to influence the immune system, it will be interesting to include characterization of the microbiome in future studies focusing on fatigue in IBD or even consider testing therapeutic microbes for treatment of IBD-related fatigue. . $^{41,48}$

\section{Conclusion}

We show for the first time that differences in immune parameters are associated with fatigue symptoms in patients with IBD without clinically active disease. These data warrant further investigation into the possible causal relations between these parameters and the fatigue symptoms since this may lead to an effective resolution in part of the patients with IBD with fatigue.

\section{Disclosure}

The authors report no conflicts of interest in this work. 


\section{References}

1. Minderhoud IM, Samsom M, Oldenburg B. Crohn's disease, fatigue, and infliximab: is there a role for cytokines in the pathogenesis of fatigue? World J Gastroenterol. 2007;13(14):2089-2093.

2. Jelsness-Jorgensen LP, Bernklev T, Henriksen M, Torp R, Moum BA. Chronic fatigue is associated with impaired health-related quality of life in inflammatory bowel disease. Aliment Pharmacol Ther. 2011;33(1): $106-114$

3. Romberg-Camps MJ, Bol Y, Dagnelie PC, et al. Fatigue and healthrelated quality of life in inflammatory bowel disease: results from a population-based study in the Netherlands: the IBD-South Limburg cohort. Inflamm Bowel Dis. 2010;16(12):2137-2147.

4. Graff LA, Vincent N, Walker JR, et al. A population-based study of fatigue and sleep difficulties in inflammatory bowel disease. Inflamm Bowel Dis. 2011;17(9):1882-1889.

5. Vogelaar L, Van't Spijker A, van Tilburg AJ, Kuipers EJ, Timman R, van der Woude CJ. Determinants of fatigue in Crohn's disease patients. Eur J Gastroenterol Hepatol. 2013;25(2):246-251.

6. Dantzer R, O'Connor JC, Freund GG, Johnson RW, Kelley KW. From inflammation to sickness and depression: when the immune system subjugates the brain. Nat Rev Neurosci. 2008;9:46-56.

7. de Jonge WJ. The gut's little brain in control of intestinal immunity. ISRN Gastroenterol. 2013;2013:630159.

8. Simren M, Svedlund J, Posserud I, Bjornsson ES, Abrahamsson H. Predictors of subjective fatigue in chronic gastrointestinal disease. Aliment Pharmacol Ther. 2008;28(5):638-647.

9. Kavelaars A, Kuis W, Knook L, Sinnema G, Heijnen CJ. Disturbed neuroendocrine-immune interactions in chronic fatigue syndrome. J Clin Endocrinol Metab. 2000;85(2):692-696.

10. Fletcher MA, Zeng XR, Barnes Z, Levis S, Klimas NG. Plasma cytokines in women with chronic fatigue syndrome. JTransl Med. 2009;7:96.

11. Patarca R. Cytokines and chronic fatigue syndrome. Ann NY Acad Sci. 2001;933:185-200.

12. Gaab J, Rohleder N, Heitz V, et al. Stress-induced changes in LPSinduced pro-inflammatory cytokine production in chronic fatigue syndrome. Psychoneuroendocrinology. 2005;30(2):188-198.

13. ter Wolbeek M, van Doornen LJ, Kavelaars A, van de Putte EM, Schedlowski M, Heijnen CJ. Longitudinal analysis of pro- and antiinflammatory cytokine production in severely fatigued adolescents Brain Behav Immun. 2007;21(8):1063-1074.

14. Dantzer R. Cytokine-induced sickness behavior: mechanisms and implications. Ann NY Acad Sci. 2001;933:222-234.

15. Skowera A, Cleare A, Blair D, Bevis L, Wessely SC, Peakman M. High levels of type 2 cytokine-producing cells in chronic fatigue syndrome. Clin Exp Immunol. 2004;135(2):294-302.

16. Bonaz BL, Bernstein CN. Brain-gut interactions in inflammatory bowel disease. Gastroenterology. 2013;144(1):36-49.

17. van der Zanden EP, Snoek SA, Heinsbroek SE, et al. Vagus nerve activity augments intestinal macrophage phagocytosis via nicotinic acetylcholine receptor alpha4beta2. Gastroenterology. 2009;137(3):e1-e4.

18. Morris G, Anderson G, Galecki P, Berk M, Maes M. A narrative review on the similarities and dissimilarities between myalgic encephalomyelitis/chronic fatigue syndrome (ME/CFS) and sickness behavior. BMC Med. 2013;11:64.

19. Heesen C, Nawrath L, Reich C, Bauer N, Schulz KH, Gold SM. Fatigue in multiple sclerosis: an example of cytokine mediated sickness behaviour? J Neurol Neurosurg Psychiatry. 2006;77(1):34-39.

20. Bower JE, Ganz PA, Aziz N, Fahey JL. Fatigue and proinflammatory cytokine activity in breast cancer survivors. Psychosom Med. 2002;64(4) 604-611.

21. Bower JE, Ganz PA, Aziz N, Olmstead R, Irwin MR, Cole SW. Inflammatory responses to psychological stress in fatigued breast cancer survivors: relationship to glucocorticoids. Brain Behav Immun. 2007;21(3):251-258.

22. Bower JE, Lamkin DM. Inflammation and cancer-related fatigue: mechanisms, contributing factors, and treatment implications. Brain Behav Immun. 2013;30(suppl):S48-S57.
23. Bower JE. Cancer-related fatigue: links with inflammation in cancer patients and survivors. Brain Behav Immun. 2007;21(7):863-871.

24. Vogelaar L, van't Spijker A, Timman R, et al. Fatigue management in patients with IBD: a randomised controlled trial. Gut. 2014;63(6):911-918.

25. Vercoulen JH, Alberts M, Bleijenberg G. De checklist individual strength (CIS). Gedragtherapie. 1999;32:131-136.

26. Harvey RF, Bradshaw JM. A simple index of Crohn's-disease activity. Lancet. 1980;1(8167):514.

27. Lichtiger S, Present DH, Kornbluth A, et al. Cyclosporine in severe ulcerative colitis refractory to steroid therapy. $N$ Engl J Med. 1994;330(26): 1841-1845.

28. Satsangi J, Silverberg MS, Vermeire S, Colombel JF. The Montreal classification of inflammatory bowel disease: controversies, consensus, and implications. Gut. 2006;55(6):749-753.

29. Tobin J. Estimation of relationships for limited dependent variables. Econometrica. 1958;26:24-36.

30. Penttila IA, Harris RJ, Storm P, Haynes D, Worswick DA, Marmion BP. Cytokine dysregulation in the post-Q-fever fatigue syndrome. QJM. 1998;91(8):549-560.

31. Chapenko S, Krumina A, Logina I, et al. Association of active human herpesvirus-6, -7 and parvovirus b19 infection with clinical outcomes in patients with myalgic encephalomyelitis/chronic fatigue syndrome. Adv Virol. 2012;2012:205085.

32. Fung FY, Li M, Breunis H, Timilshina N, Minden MD, Alibhai SM. Correlation between cytokine levels and changes in fatigue and quality of life in patients with acute myeloid leukemia. Leuk Res. 2013; 37(3):274-279.

33. Bower JE, Ganz PA, Irwin MR, Castellon S, Arevalo J, Cole SW. Cytokine genetic variations and fatigue among patients with breast cancer. J Clin Oncol. 2013;31(13):1656-1661.

34. Zick SM, Zwickey H, Wood L, et al. Preliminary differences in peripheral immune markers and brain metabolites between fatigued and non-fatigued breast cancer survivors: a pilot study. Brain Imaging Behav. 2014;8(4):506-516.

35. De Sanctis V, Agolli L, Visco V, et al. Cytokines, fatigue, and cutaneous erythema in early stage breast cancer patients receiving adjuvant radiation therapy. Biomed Res Int. 2014;2014:523568.

36. Minton O, Coulton GR, Stone P. Multi-analyte profiling and pathway analysis of plasma for proteins associated with cancer-related fatigue syndrome in disease-free breast cancer patients after primary treatment. BMJ Support Palliat Care. 2014;4(4):349-356.

37. Yonkers NL, Sieg S, Rodriguez B, Anthony DD. Reduced naive CD4 $\mathrm{T}$ cell numbers and impaired induction of CD27 in response to T cell receptor stimulation reflect a state of immune activation in chronic hepatitis C virus infection. J Infect Dis. 2011;203(5):635-645.

38. Bansal AS, Bradley AS, Bishop KN, Kiani-Alikhan S, Ford B. Chronic fatigue syndrome, the immune system and viral infection. Brain Behav Immun. 2012;26(1):24-31.

39. Olson NC, Doyle MF, Jenny NS, et al. Decreased naive and increased memory CD4(+) T cells are associated with subclinical atherosclerosis: the multi-ethnic study of atherosclerosis. PLoS One. 2013;8(8):e71498.

40. Brenu EW, van Driel ML, Staines DR, et al. Immunological abnormalities as potential biomarkers in chronic fatigue syndrome/myalgic encephalomyelitis. J Transl Med. 2011;9:81.

41. Groeger D, O’Mahony L, Murphy EF, et al. Bifidobacterium infantis 35624 modulates host inflammatory processes beyond the gut. Gut Microbes. 2013;4(4):325-339.

42. Maes M, Ringel K, Kubera M, Berk M, Rybakowski J. Increased autoimmune activity against 5-HT: a key component of depression that is associated with inflammation and activation of cell-mediated immunity, and with severity and staging of depression. J Affect Disord. 2012; 136(3):386-392.

43. Hardcastle SL, Brenu EW, Johnston S, et al. Serum immune proteins in moderate and severe chronic fatigue syndrome/myalgic encephalomyelitis patients. Int J Med Sci. 2015;12(10):764-772.

44. Chia J, Chia A, Voeller M, Lee T, Chang R. Acute enterovirus infection followed by myalgic encephalomyelitis/chronic fatigue syndrome (ME/ CFS) and viral persistence. J Clin Pathol. 2010;63(2):165-168. 
45. Baars JE, Nuij VJ, Oldenburg B, Kuipers EJ, van der Woude CJ. Majority of patients with inflammatory bowel disease in clinical remission have mucosal inflammation. Inflamm Bowel Dis. 2012;18(9):1634-1640.

46. Maes M, Coucke F, Leunis JC. Normalization of the increased translocation of endotoxin from gram negative enterobacteria (leaky gut) is accompanied by a remission of chronic fatigue syndrome. Neuro Endocrinol Lett. 2007;28(6):739-744.
47. Maes M, Leunis JC. Normalization of leaky gut in chronic fatigue syndrome (CFS) is accompanied by a clinical improvement: effects of age, duration of illness and the translocation of LPS from gram-negative bacteria. Neuro Endocrinol Lett. 2008;29(6):902-910.

48. Rooks MG, Veiga P, Wardwell-Scott LH, et al. Gut microbiome composition and function in experimental colitis during active disease and treatment-induced remission. ISME J. 2014;8(7):1403-1417.
Clinical and Experimental Gastroenterology

\section{Publish your work in this journal}

Clinical and Experimental Gastroenterology is an international, peerreviewed, open access, online journal publishing original research, reports, editorials, reviews and commentaries on all aspects of gastroenterology in the clinic and laboratory. This journal is included on PubMed. The manuscript management system is completely online
Dovepress

and includes a very quick and fair peer-review system, which is all easy to use. Visit http://www.dovepress.com/testimonials.php to read real quotes from published authors. 